\title{
Both sides of the same coin: Rac1 splicing regulation by EGF signaling
}

Cell Research (2017) 27:455-456. doi:10.1038/cr.2017.19; published online 10 February 2017

EGF, a well-studied mitogen for cancer cells, is revealed to induce an E3 ubiquitin ligase adaptor SPSB1, which recruits the Elongin $\mathrm{B} / \mathrm{C}$-Collin complex to trigger ubiquitylation of the negative splicing regulator hnRNP A1. This event is synergized with EGF-activated SR proteins to alter alternative splicing of a key small GTPase Rac1 to enhance cell migration, highlighting converging EGF signals on both negative and positive splicing regulators to jointly promote a key cancer pathway.

It has been long established that Epithelial Growth Factor (EGF) binds the EGF receptor (EGFR) to activate several cascades of signaling events to promote cell proliferation, which has been linked to a variety of cancers in humans [1]. Cell migration is a key event for cancer metastasis, which is a highly orchestrated multistep process that involves membrane ruffling and lamellipodial protrusion [2]. Rho GTPases play important roles in this process, one of which is Rac1. Rac1 has been shown to be required for EGF-induced cell migration and one mechanism is the induction of a Rac1 GTP exchange factor (GEF) Tiam1, which activates Rac1 in response to EGF-induced activation of Akt [3]. Interestingly, Rac1 is also known to undergo alternative splicing by including an alternative exon $3 \mathrm{~b}$ to generate Rac1b, as illustrated in Figure $1 \mathrm{~A}$, and the insertion of 19 amino acids from this alternative exon arrests Rac $1 b$ in the GTP-bound form [4].

Alternative pre-mRNA splicing is a form of post-transcriptional regulation of gene expression, which expands the proteome in mammalian cells and has been linked to a variety of developmental and disease processes [5]. In the case of Rac1 splicing, early studies have established promotion of the alternative exon $3 b$ by the SR protein SRSF1 [6], and repression of the alternative exon by hnRNP A1 [7], which has been a common theme in the regulation of alternative splicing by competing RNA-binding proteins (RBPs) [8]. The question is how these regulatory events are connected to EGF signaling. In fact, EGF has been shown to induce a wide range of alternative splicing events via the Akt-SRPK-SR axis in which EGF activates SR protein-specific kinases (SRPK1 and 2) via Akt and the SR kinases in turn phosphorylate and activate SR proteins [9], a signaling cascade that has been linked to tumorigenesis in both animal models and humans [10]. Hui and colleagues have now explored EGF signaling to hnRNP A1, establishing the mechanism for inactivation of this negative splicing regulator by polyubiquitylation [11].

To study EGF-regulated splicing, the authors first set out to use unbiased RNA-seq to identify altered splicing events, leading to the identification of several hundred EGF-induced alternative splicing events. As alternative splicing has been well known to subject to regulation by various RBPs, each with a degree of RNA binding specificity, the authors performed motif analysis of EGF-induced splicing events. One of the significantly enriched motifs appears to correspond to the binding site for
hnRNP A1, one of the best characterized splicing regulators in mammalian cells, which is to antagonize the SR family of splicing factors in splice site recognition [8].

As the total hnRNP A1 level appears unchanged in EGF-treated cells, the authors explored its post-transcriptional modification, and found that hnRNP A1 underwent polyubiquitylation upon EGF treatment. This important clue led the authors to pursue the specific E3 ligase(s) involved. Through an unbiased screen on a panel of proteins involved in ubinquitylation, they identified SPSB1, an adaptor for the Elongin B/C-Cul5 E3 ligase. SPSB1 was dramatically induced in EGF-treated cells. A series of elegant biochemical studies then demonstrated that SPSB1 bridged the Elongin B/C E3 ligase to catalyze polyubiquitylation of hnRNP A1 on Lys 183 and Lys293 residues. Interestingly, this unusual K29linked polyubiquitylation did not seem to alter the protein level of hnRNP A1, but appeared to have switched a fraction of hnRNP A1 to the cytoplasm. More importantly, the modified hnRNP A1 showed reduced RNA binding affinity in the nucleus, as established by RIP (RNA ImmunoPrecipitation-PCR). This study has thus established a pathway for EGFinduced inactivation of hnRNP A1.

To attribute this newly elucidated pathway to cell migration, the authors discovered a key role of hnRNP A1 in suppressing the inclusion of the Rac1 alternative exon $3 b$. By directly blocking this alternative splicing event with an antisense oligonucleotide, the authors demonstrated a vital contribution of 
A

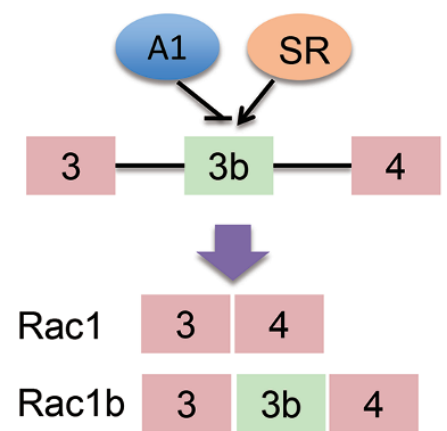

B

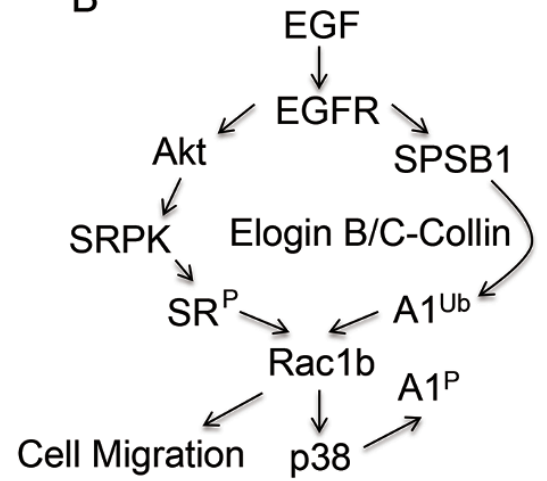

Figure 1 Cell migration induced by EGF via regulated alternative splicing of the Rho GTPase Rac1. (A) Alternative splicing of Rac1 is positively regulated by SR proteins and negatively regulated by hnRNP A1. The inclusion or skipping of the alternative exon $3 b$ generates Rac1 or Rac1b, the latter of which is arrested in the GTP-bound form. (B) EGF signaling to modulate the activities of key splicing regulators. Via the Akt pathway, EGF activates SR protein kinases (SRPK1/2), which in turn phosphorylate and activate SR proteins to induce Rac1b splicing. The present study by Hui and colleagues demonstrates that EGF also induces the E3 ligase adaptor SPSB1, which facilitates hnRNP A1 polyubiquitylation by the Elogin B/CCollin E3 ligase. This modification attenuates RNA binding of hnRNP A1, thereby de-repressing Rac1b splicing. Based on the literature information, it is possible that Rac1b further activates the stress kinase $\mathrm{p} 38$, which phosphorylates hnRNP A1, resulting in the accumulation of a fraction of hnRNP A1 in the cytoplasm. This cellular restriction may also contribute to de-repressed Rac1b splicing.

Rac1b to EGF-induced cell migration. Interestingly, inactivation of hnRNP A1 by EGF-induced polyubiquitylation partially contributed to Rac1b splicing. Noticing that the activated SRPK pathway also induced Rac $1 \mathrm{~b}$ splicing from a previous study [9], the authors went on to demonstrate that the activated SRPKSR pathway was indeed synergistic with inactivated hnRNP A1 to fully induce Rac1 splicing to produce the Rac $1 \mathrm{~b}$ isoform. As illustrated in Figure 1B, this study is the first to reveal that a signaling pathway simultaneously activates SR proteins by phosphorylation and inactivates hnRNP A1 by polyubiquitylation to achieve synergistic effects on Rac1 splicing to promote cell migration.

There are a few unsolved questions in the present study. One is the induction of SPSB1 by EGF. As enhanced cell migration of tumor cells is often a consequence of epithelial-mesenchymal transition (EMT), which can also be induced by TGF $\beta$, a previous study showed that TGF $\beta$ dramatically induced the expression of SPSB1 at the RNA level, indicative of a transcriptional mechanism [12]. Therefore, it is likely that EGF treatment might have induced SPSB1 gene expression. The present study also provides evidence that the mutations in the ubiqintylation sites in hnRNP A1 switched a fraction of this RBP to the cytoplasm, but it is unclear whether this is a direct or indirect effect. In fact, a previous study demonstrated that stress-activated p38 dramatically induced hnRNPA1 to accumulate in the cytoplasm [13]. Interestingly, activated Rac1 has been shown to activate p38, which can feedback EGFR activation
[14], and activated p38 appears to play an essential role in enhancing TGF $\beta$ induced cell migration [15]. Considered together, EGF-induced Rac1b may activate $\mathrm{p} 38$, which may in turn phosphorylate hnRNP A1 to cause its accumulation in the cytoplasm, as indicated in Figure 1B. While this potential pathway will require further investigation, it appears that EGF mobilizes a large village to accomplish regulated splicing during tumor progression.

\section{Xiang-Dong $\mathrm{Fu}^{1}$}

${ }^{1}$ Department of Cellular and Molecular Medicine, University of California, San Diego, La Jolla, CA 92093-0651, USA

Correspondence: Xiang-Dong Fu

E-mail: xdfu@ucsd.edu

\section{References}

1 Avraham R, Yarden Y. Nat Rev Mol Cell Biol 2011; 12:104-117.

2 Ridley AJ, Schwartz MA, Burridge K, et al. Science 2003; 302:1704-1709.

3 Zhu G, Fan Z, Ding M, et al. Oncogene 2015; 34:5971-5982.

4 Fiegen D, Haeusler LC, Blumenstein L, et al. J Biol Chem 2004; 279:4743-4749.

5 Scotti MM, Swanson MS. Nat Rev Genet 2016; 17:19-32.

6 Goncalves V, Matos P, Jordan P. Hum Mol Genet 2009; 18:3696-3707.

7 Pelisch F, Khauv D, Risso G, et al. J Cell Biochem 2012; 113:2319-2329.

8 Fu XD, Ares M Jr. Nat Rev Genet 2014; 15:689-701.

9 Zhou Z, Qiu J, Liu W, et al. Mol Cell 2012; 47:422-433.

10 Wang P, Zhou Z, Hu A, et al. Mol Cell 2014; 54:378-391.

11 Wang F, Fu X, Chen F, et al. Cell Res 2017; 27:540-558.

12 Liu S, Nheu T, Luwor R, et al. J Biol Chem 2015; 290:17894-17908.

13 van der Houven van Oordt W, Diaz-Meco MT, Lozano J, et al. J Cell Biol 2000; 149:307-316.

14 Cheng H, Kartenbeck J, Kabsch K, et al. J Cell Physiol 2002; 192:234-243.

15 Bakin AV, Rinehart C, Tomlinson AK, et al. $J$ Cell Sci 2002; 115:3193-3206. 